\title{
The impact of mobile virtual reality-enhanced relaxation training on anxiety levels in patients undergoing cardiac rehabilitation
}

\author{
Justyna Maciołek ${ }^{1}$, Wojciech Wąsek², Bogumił Kamiński ${ }^{3}$, Katarzyna Piotrowicz ${ }^{1}$, Paweł Krzesiński ${ }^{1}$ \\ 1 Department of Cardiology and Internal Diseases, Military Institute of Medicine, Warsaw, Poland \\ 2 Department of Internal Medicine, Institute of Medical Sciences, College of Medical Sciences, University of Rzeszów, Rzeszów, Poland \\ 3 Decision Analysis and Support Unit, SGH Warsaw School of Economics, Warsaw, Poland
}

Correspondence to: Justyna Maciołek, MSc, Department of Cardiology and Internal Diseases, Military Institute of Medicine, ul. Szaserów 128, 04-141 Warszawa, Poland, phone: +48261817285 , email: jmaciolek@wim.mil.pl Received: April 7, 2020 Revision accepted: July 15, 2020 Published online: July 24, 2020. Kardiol Pol. 2020; 78 (10): 1032-1034 doi:10.33963/KP.15528

Copyright by the Author(s), 2020
Introduction The role of a psychologist in the cardiac rehabilitation ward includes undertaking actions aimed to reduce psychological stress, known to have a negative impact on patient prognosis. Available data indicate that anxiety may increase the risk of developing heart diseases and death. ${ }^{1}$ The observations stimulate search for effective stress-reduction methods. In a study by Dixhoorn and Duivenvoorden, ${ }^{2}$ relaxation training achieved a 31\% reduction in hospitalizations as compared with the control group.

Enhancing relaxation training with virtual reality (VR) technologies is encouraged by reports confirming the efficacy of this method in reducing pain and stress levels in patients following cardiac surgery ${ }^{3}$ and anxiety levels in post-trauma patients. ${ }^{4}$

The main objective of the study was to analyze the impact of relaxation training enhanced by mobile VR on anxiety levels in patients undergoing cardiac rehabilitation.

Methods The study included 65 patients (44 men [68\%] and 21 women [32\%]) at the mean (SD) age of 59.8 (11.8) years. A total of 20 patients (30.8\%) were active smokers or abstainers since cardiac event, in NYHA class I or II, undergoing the second stage of cardiac stationary rehabilitation; 56 patients had a history of angioplasty (39 due to myocardial infarction), 6 had prior coronary bypass surgery, and 3 were hospitalized due to heart failure. Exclusion criteria included cognitive or somatic deficits prohibiting participation in paper-and-pencil tests and/or the use of VR equipment. Patients were assigned successively and alternately, based on admission time, to the experimental group (32 patients, 22 men [69\%]) and the control group (33 patients, 22 men [67\%]). Each participant was informed about the goal of the study and provided written consent. The study report was approved by the Bioethical Committee of the Military Institute of Medicine (no. 11/WIM/2017).

Procedure At the beginning of the rehabilitation process (days 1-3), each patient filled out the State-Trait Anxiety Inventory (STAI). Persons assigned to the experimental group participated in six 20-minute relaxation training sessions, which utilized virtual reality technologies, provided via a mobile application (individually, under psychologist's supervision, every second working day). Patients in the control group took part in six 20-minute relaxation training group sessions, conducted every second working day by a psychologist. During the last session, the participants completed the STAI questionnaires again. All patients received psychoeducation on stress.

In the experimental group, VR-enhanced training was conducted with the use of an Apple iPhone 5S (Apple, Cupertino, California, United States). Exposure to VR was provided via Google Cardboard-type VR glasses (Primark, Dublin, Ireland), with the use of an application by RelaxVR (San Francisco, California, United States), which generates a natural virtual landscape model accompanied by relaxation music, provided by the software. Mobile-type intervention was chosen based on its potential to be 
TABLE 1 Comparison of the intensity of anxiety-state and anxiety-trait before and after the relaxation training (paired $t$ test)

\begin{tabular}{llll} 
Parameter & Before & After & $P$ value \\
\hline Anxiety-trait & & & \\
\hline Experimental group & $43.31(9.75)$ & $40.59(7.16)$ & 0.01 \\
\hline Control group & $43.34(9.95)$ & $38.36(9.09)$ & 0.001 \\
\hline Anxiety-state & & \\
\hline Experimental group & $39.94(9.15)$ & $35.91(8.01)$ & 0.01 \\
\hline Control group & $37.39(8.75)$ & $36.39(8.45)$ & 0.38 \\
\hline
\end{tabular}

Data are presented as mean (SD).

used by patients at any place and time, thus increasing its accessibility to a large group of recipients. ${ }^{5}$ Each patient was sitting on a rotating chair, allowing them to move as reflected in the VR application.

Patients from the control group participated in relaxation sessions conducted by a psychologist which included visualisations with elements of the autogenic training, accompanied by relaxation music from the RelaxVR application.

Diagnostic tool The State-Trait Anxiety Inventory (STAI) is a diagnostic tool designed to study anxiety perceived as a state and as a trait.

Statistical analysis The results were subjected to statistical analysis using the R 3.5.1. software suite (The R Foundation for Statistical Computing, Vienna, Austria). Continuous variables were presented as means (SD) and categorical variables as numbers and percentages. The paired $t$ test was applied to compare continuous variables between groups as a basic testing procedure for all tests comparing average values. The Shapiro-Wilk test was used to test for normality of distribution of anxiety-trait, anxiety-state, and age variables in both groups and null hypothesis was not rejected. The $\mathrm{X}^{2}$ was used to compare nominal variables. A $P$ value equal to or less than 0.01 was considered significant.

Results and discussion On admission, there were no differences between the groups with regard to anxiety-state $(P=0.26)$, anxiety-trait $(P=0.99)$, age $(P=0.09)$, sex $(P=0.86)$, and smoking status $(P=0.65)$. After the treatment, there was a reduction in the mean intensity of the anxiety-trait indicator in both groups (experimental group, $P=0.01$ and control group, $P=0.001$ ). There was a reduction in the mean anxiety-state in the experimental group $(P=0.01)$ as opposed to the control group $(P=0.38)$. Data are shown in TABLE1.

The use of mobile VR in relaxation training administered to patients undergoing cardiac rehabilitation was shown to be an effective method of reducing stress. Of note, there was no decrease in the anxiety-state level in the control group, particularly in the context of a meta-analysis that demonstrated that relaxation training reduced anxiety-state levels. ${ }^{6}$ The meta-analysis, however, indicated lower effectiveness in patients with advanced age and those with somatic diseases. ${ }^{7}$ It is possible that patients from the control group did not follow the instructions, which in turn eliminated the potential benefits of the training in reducing anxiety.

Reduction in the level of the anxiety-trait is also worth noting. It is by definition a constant behavioral disposition; however, recent data suggest anxiety-trait measured by the STAI might also be a measure of a negative affect, and hence, may be a modifiable feature. ${ }^{8}$

The effect of VR-supported relaxation can be compared to the effects of mindful meditation. Both utilize focused attention and observing. However, relaxation training led by a psychologist instructs participants to deliberately relax, and mindfulness and VR training emphasize awareness without any attempt to change their emotions. Relaxation and mindfulness involve a different neural mechanism ${ }^{9}$; whether it is also the case in VR-enhanced trainings requires further studies.

A reduction of the anxiety-trait level in VR-enhanced relaxation training may be of particular importance in survivors of acute coronary syndrome (ACS) with D-type personalities, that is, those who tend to succumb to negative emotions, including strong feelings of anxiety. This group displays a tendency to maintain long-term increased anxiety levels following ACS..$^{10}$ Such negative effect, increased in the course of the first weeks after ACS, is crucial in patients who decide to resign from cardiac rehabilitation. ${ }^{11}$

Our results give hope of providing effective assistance to cardiac patients as anxiety might have an impact on the course of cardiac rehabilitation ${ }^{12}$ and testing..$^{13}$ Further research into the application of VR-enhanced trainings is needed. 


\section{ARTICLE INFORMATION}

CONFLICT OF INTEREST None declared

OPEN ACCESS This is an Open Access article distributed under the terms of the Creative Commons Attribution-NonCommercial-NoDerivatives $4.0 \mathrm{In}$ ternational License (CC BY-NC-ND 4.0), allowing third parties to download articles and share them with others, provided the original work is properly cited, not changed in any way, distributed under the same license, and used for noncommercial purposes only. For commercial use, please contact the journal office at kardiologiapolska@ptkardio.pl.

HOW TO CITE MaciołekJ, Wąsek W, Kamiński B, et al. The impact of mobile virtual reality-enhanced relaxation training on anxiety levels in patients undergoing cardiac rehabilitation. Kardiol Pol. 2020; 78: 1032-1034. doi:10.33963/ KP.15528

\section{REFERENCES}

1 Gallagher J, Parenti G, Doyle F. Psychological aspects of cardiac care and rehabilitation: time to wake up to sleep? Curr Cardiol Rep. 2015; 17: 111-124.

2 van Dixhoorn J], Duivenvoorden HJ. Effect of relaxation therapy on cardiac events after myocardial infarction: a 5-year follow-up study. J Cardiopulm Rehabil. 1999; 19: 178-185.

3 Mosso-Vázquez JL, Gao K, Wiederhold BK, Wiederhold MD. Virtual reality for pain management in cardiac surgery. Cyberpsychol Behav Soc Netw. 2014; 17: 371-378.

4 Wiederhold BK, Wiederhold MD. Virtual reality treatment of posttraumatic stress disorder due to motor vehicle accident. Cyberpsychol Behav Soc Netw. 2010; 13: 21-27.

5 Wiederhold BK, Gao K, Kong L, Wiederhold MD. Mobile devices as adjunctive pain management tools. Cyberpsychol Behav Soc Netw. 2014; 17: 385-389.

6 van Dixhoorn J, White A. Relaxation therapy for rehabilitation and prevention in ischaemic heart disease: a systematic review and meta-analysis. Eur J Cardiovasc Prev Rehabil. 2005; 12: 193-202.

7 Manzoni GM, Pagnini F, Castelnuovo G, Molinari E. Relaxation training for anxiety: a ten-years systematic review with meta-analysis. BMC Psychiatry. 2008; 2 : 41-52.

8 Balsamo, M, Romanelli, R, Innamorati, M, et al. The state-trait anxiety inventory: shadows and lights on its construct validity. J Psychopathol Behav Assess. 2013; 35: 475-486.

9 Sevinc G, Hölzel BK, Hashmi J, et al. Common and dissociable neural activity after mindfulness-based stress reduction and relaxation response programs. Psychosom Med. 2018; 80: 439-451.

10 Versteeg $\mathrm{H}$, Roest AM, Denollet J. Persistent and fluctuating anxiety levels in the 18 months following acute myocardial infarction: the role of personality. Gen Hosp Psychiatry. 2015; 37: 1-6.

11 Jones MC, Smith K, Herber 0 , et al. Intention, beliefs and mood assessed using electronic diaries predicts attendance at cardiac rehabilitation: an observational study. Int J Nurs Stud. 2018; 88: 143-152.

12 Rao A, Zecchin R, Newton PJ, et al. The prevalence and impact of depression and anxiety in cardiac rehabilitation: a longitudinal cohort study. Eur J Prev Cardiol. 2020; 27: 478-489.

13 Smarż K, Jaxa-Chamiec T, Chwyczko T, et al. Cardiopulmonary exercise testing in adult cardiology: expert opinion of the Working Group of Cardiac Rehabilitation and Exercise Physiology of the Polish Cardiac Society. Kardiol Pol. 2019; 77: $730-756$ 